\title{
Real-Time-Monitoring in der Behandlung von Zwangsstörungen: Technologie und Fallbeispiel
}

\author{
Igor Tominschek ${ }^{a} \quad$ Günter Schiepek ${ }^{b, c} \quad$ Claudia Mehl $^{a} \quad$ Kristina Maier $^{a} \quad$ Stephan Heinzel ${ }^{a, b}$ \\ Christoph Bauhofer ${ }^{d}$ Benjamin Berbic ${ }^{d} \quad$ Michael Zaudiga, e \\ a Tagklinik Westend / München, Deutschland \\ ${ }^{b}$ Medizinische Privatuniversität Paracelsus, Salzburg, Österreich, \\ ${ }^{c}$ Institut für Psychologie, Ludwig-Maximilians-Universität München, \\ dCenter for Complex Systems, Ostfildern, Deutschland \\ e Psychosomatische Klinik Windach, Deutschland
}

\section{Schlüsselwörter}

Real-Time Monitoring · Prozessfeedback .

Zwangsstörung

\section{Zusammenfassung}

Hintergrund: Dargestellt wird die Möglichkeit, Behandlungsverläufe mit Hilfe von Internet-basierten Fragebögen abzubilden und zu analysieren. Tägliche Selbsteinschätzungen des Patienten erlauben es, ein detailliertes Bild des Therapieverlaufs darzustellen und für Interventionsentscheidungen zu nutzen. Patient und Methoden: Bei dem in diesem Beitrag exemplarisch ausgewählten Patienten lagen eine Zwangsstörung und eine komorbide depressive Episode vor, die in einer psychosomatischen Tagesklinik behandelt wurden. Ergebnis: Quantitative Zeitreihen und qualitative Angabe im Rahmen des Internet-basierten Tagebuchs spiegeln wichtige Phasen im Veränderungsprozess wider. Schlussfolgerungen: Die EDV-basierte Auswertung der Fragebögen unmittelbar nach Eingabe der Antworten durch den Patienten ermöglicht ein Behandlungsfeedback für Patient und Therapeut, steigert die Transparenz des Therapieverlaufs, unterstützt das Selbstwirksamkeitserleben der Patienten und generiert ohne Zusatzaufwand eine Prozess-Outcome-Dokumentation.

\author{
Key Words \\ Computer-assisted Real-Time Monitoring . \\ Feedback-controlled therapy . \\ Obsessive-compulsive disorder
}

\section{Summary}

Real-Time-Monitoring in the Treatment Obsessive-

Compulsive Disorders: Technology and Case Report

Background: New methods of internet-based real-time monitoring of psychotherapy processes allow for the documentation, analysis, and data-driven control of behaviour therapy. Daily ratings of the patient result in highly resolved time series of the change dynamics. Method and Patient: The Synergetic Navigation System was used in the treatment setting of a day hospital. This method is specialized in the representation of critical instabilities and pattern transitions of the process. The patient of this case report had the combined diagnosis of obsessive-compulsive disorder and depressive episodes. Result: Quantitative time-series and qualitative data from the internet-assisted diaries mirror important features of the change process. Conclusion: The method could help optimise treatment effects of behavioural interventions by using the feedback on necessary conditions, appropriate timing, and short-term effects of these interventions. Patients' motivation for change can be enhanced and the navigation system realises the continuous documentation of treatment processes and effects.

\begin{tabular}{ll}
\hline KARGER & @ 2008 S. Karger GmbH, Freiburg \\
Fax +497614520714 & $\begin{array}{l}\text { Accessible online at: } \\
\text { www.karger.com/ver }\end{array}$ \\
Information@Karger.de \\
www.karger.com
\end{tabular}




\section{Einleitung}

Die Verbreitung des Internets macht es möglich, Fragebögen und andere Einschätzungen von Patienten online zu erheben und praktisch ohne Zeitverzögerung auszuwerten ( RealTime-Monitoring»). Diese technische Voraussetzung liefert die Grundlage für ein Monitoring, das über Fragebogenerhebungen von Patienten in der Therapiestunde hinausgeht. Dadurch sind Befragungen zwischen den Therapiestunden möglich, die genauere Einblicke in Veränderungsprozesse erlauben.

Den aktuellen Entwicklungen von Verfahren des Real-TimeMonitorings liegen mehrere Entwicklungsströmungen zugrunde, die sich für die Psychotherapie in Praxis und Forschung als bedeutsam erwiesen haben:

1. Neben den eingesetzten Behandlungsmethoden spielen Wirkfaktoren eine Rolle, die sich erst während des Behandlungsprozesses formen oder durch diesen modifiziert werden, z.B. die Qualität der Therapiebeziehung, die Veränderungsmotivation und die Compliance des Patienten, die erlebten Therapiefortschritte, die Bewertung der Behandlung durch den Patienten oder die Kooperation von Bezugspersonen im sozialen Umfeld des Patienten [Lambert und Ogles, 2004; Clarkin und Levy, 2004; Orlinsky et al., 2004].

2. Die Erfassung von psychologischen oder physiologischen Daten im Feld ist durch mobile Speicherungssysteme oder das Internet nicht nur technisch (leichter) möglich geworden, sondern erweist sich auch als mindestens ebenso valide und reliabel wie die Datenerfassung im Labor oder in artifiziellen Settings (z.B. Gedächtnis- und Bewertungsbiases). Zudem erhält man Daten, die in der Retrospektive (z.B. in einer Therapiesitzung mit Rückblick auf die Zeit seit dem letzten Termin) nicht mehr generierbar wären [Fahrenberg et al., 2002, 2007].

3. Qualitätssicherung ist eine gesetzlich wie ethisch notwendige Aufgabe. Aus Gründen der Aufwandsminimierung und Ökonomisierung greifen viele Einrichtungen daher bereits auf das Internet als Dateneingabemöglichkeit zurück. Die Daten stehen nach der Eingabe unmittelbar für weitere Auswertungen zur Verfügung und können - unter Berücksichtigung des Datenschutzes - dort abgerufen werden, wo sie gebraucht werden [Percevic et al., 2006]. Neben der Ergebnisqualität rückt zunehmend auch die Prozessqualität in den Blick, deren Verbesserung kurzfristige Feedbackschleifen erforderlich macht.

4. Die Prozess-Outcome-Forschung hat in den letzten Jahren Zeitreihendaten zur Verfügung gestellt und mit geeigneten Verfahren untersucht, welche Psychotherapie als nichtlinearen und nichtstationären Prozess ausweisen [Schiepek et al., 1997; Kowalik et al., 1997; Haken und Schiepek, 2006]. Dies bedeutet, dass sich nichtlineare dynamische Muster im Laufe des Prozesses selbst verändern und in Form von Kaskaden ineinander übergehen. Solche qualitativen Sprünge zwischen raum-zeitlichen oder funktionellen Mus- tern werden in der Physik als Phasenübergänge, in der Psychologie als Ordnungsübergänge bezeichnet. Geeignete theoretische Modelle hierfür werden von der Synergetik der Theorie der Selbstorganisation [Haken, 2004] - geliefert, welche Psychotherapie als Schaffen von spezifischen Bedingungen für neuronale, psychische und soziale Selbstorganisationsprozesse interpretiert [Haken und Schiepek, 2006]. Dieses Modell von Lernen und Entwicklung ist insofern naheliegend, als gerade das Gehirn als komplexes, nichtlineares System von Neuronen und Neuronenverbänden funktioniert [Haken, 2002, 2003; Popovych et al., 2005, 2006; Singer und Gray, 1995; Strunk und Schiepek, 2006]

Erfahrungen mit Methoden des Real-Time-Monitoring machen deutlich, dass damit effektive Behandlungsverbesserungen möglich sind [Asay et al., 2002]. Die Arbeitsgruppe um Michael J. Lambert benutzt einen Vergleich zwischen dem aktuellen Therapieverlauf eines Patienten und dem Referenzverlauf anderer Patienten gleicher Diagnose, um Abweichungen in negativer Richtung zu erkennen [Lambert et al., 2001a, b, 2002]. Dadurch konnten die Effizienz der Behandlung gesteigert und die Kosten reduziert werden.

\section{Real-Time-Monitoring mit Hilfe des Synergetic Navigation System}

Die von uns verwendete Web-Applikation ermöglicht eine Dateneingabe über einen Standard-PC mit Internetanschluss oder über Browser-fähige Handys. Die Dateneingabe, -verwaltung und -speicherung erfolgt über einen externen Server (Internet) oder einen internen Server (Intranet). Der Datenschutz entspricht dem des E-Banking. Bei wiederholten Befragungen (z.B. im Verlauf einer Psychotherapie) werden die Items jeweils randomisiert vorgegeben. Falls ein Fragebogen in der vorgesehenen Zeitspanne nicht ausgefüllt wird, kann er später nachgetragen werden. Neben der Befragungs- und Dateneingabefunktion verfügt das System über eine Patientenadministration, die sich an der Psy-BaDo [Heuft und Senf, 1998] orientiert. Erfasst werden darin neben soziodemographischen und familiären Daten die Diagnosen (ICD-10, DSM IV), der Grad körperlicher und psychischer Belastung und die Inanspruchnahme medizinischer Leistungen. Mit dem System können somit elektronische Patientenakten geführt werden. Das System hält lizenzfreie Outcome-Fragebögen zur Qualitätsdokumentation und auch Fragebögen zur Prozesserfassung bereit. Die quantitative Dateneingabe kann über gestufte Likert- oder über visuelle Analogskalen erfolgen. Beim Fragebogen zur Erfassung des Therapieverlaufs handelt es sich um leichte Abwandlungen des Therapie-Prozessbogens (TPB), wie er für den Einsatz in der Aachener Prozess-OutcomeStudie zur stationären Psychotherapie entwickelt und dort validiert sowie auf seine Gütekriterien überprüft wurde [Haken und Schiepek, 2006; Schiepek et al., 2003]. Die Faktorenstruktur gliedert sich wie folgt:

I Therapeutische Fortschritte / Zuversicht / Selbstwirksamkeit

II Klima / Atmosphäre auf der Station

III Beziehungsqualität / Offenheit / Vertrauen zu den Therapeuten

IV Dysphorische Affektivität/Innenorientierung

V Perspektivenerweiterung/Innovation

VI Intensität der Problembearbeitung

VII Symptom- und Problembelastung

Im hier vorliegenden Kontext einer Tagesklinik wurde Faktor II aufgespalten in Items, die sich auf das soziale Klima an der Tagesklinik beziehen (IIa), und in solche, die sich auf das soziale Klima zuhause und im natürlichen Umfeld des Patienten beziehen (IIb). Dies ist eine wichtige 

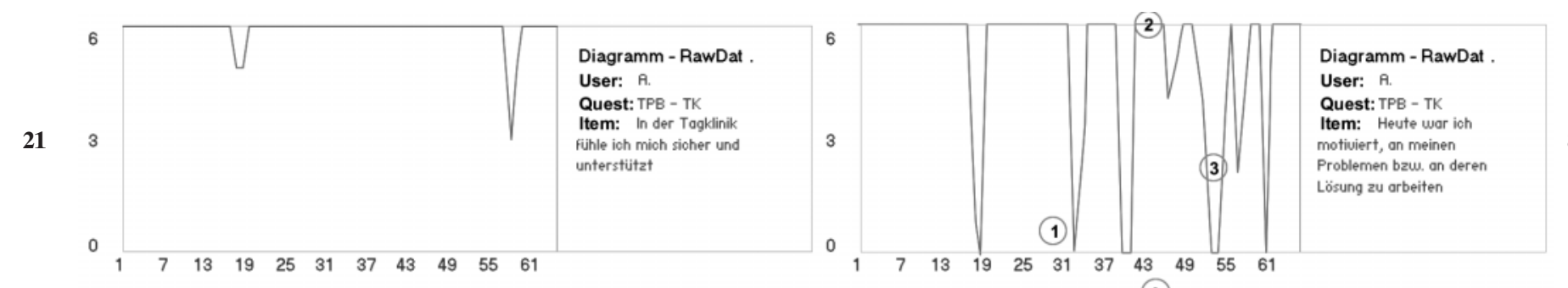

(2)
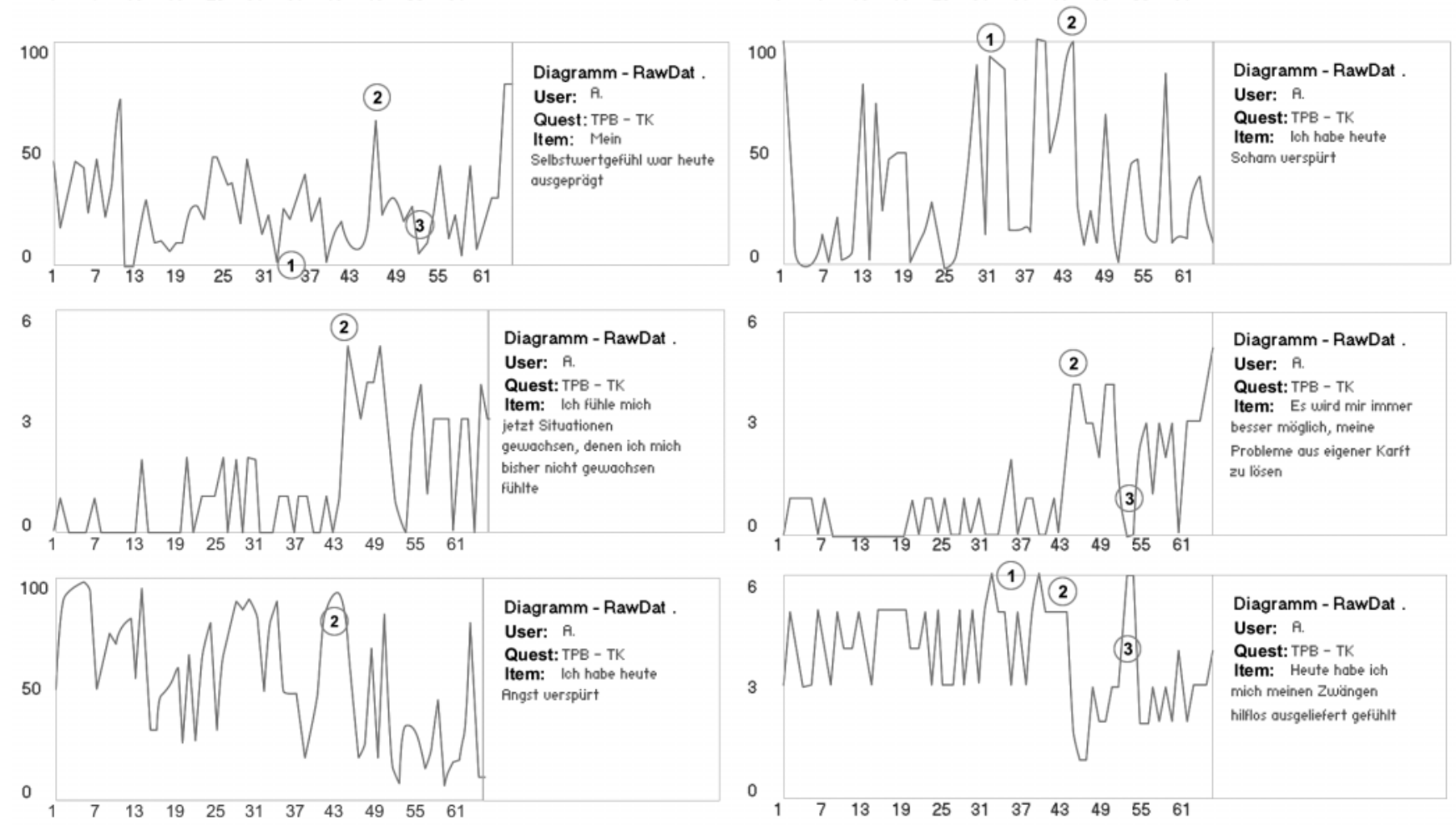

$\begin{array}{lllllllllll}1 & 7 & 13 & 19 & 25 & 31 & 37 & 43 & 49 & 55 & 61\end{array}$
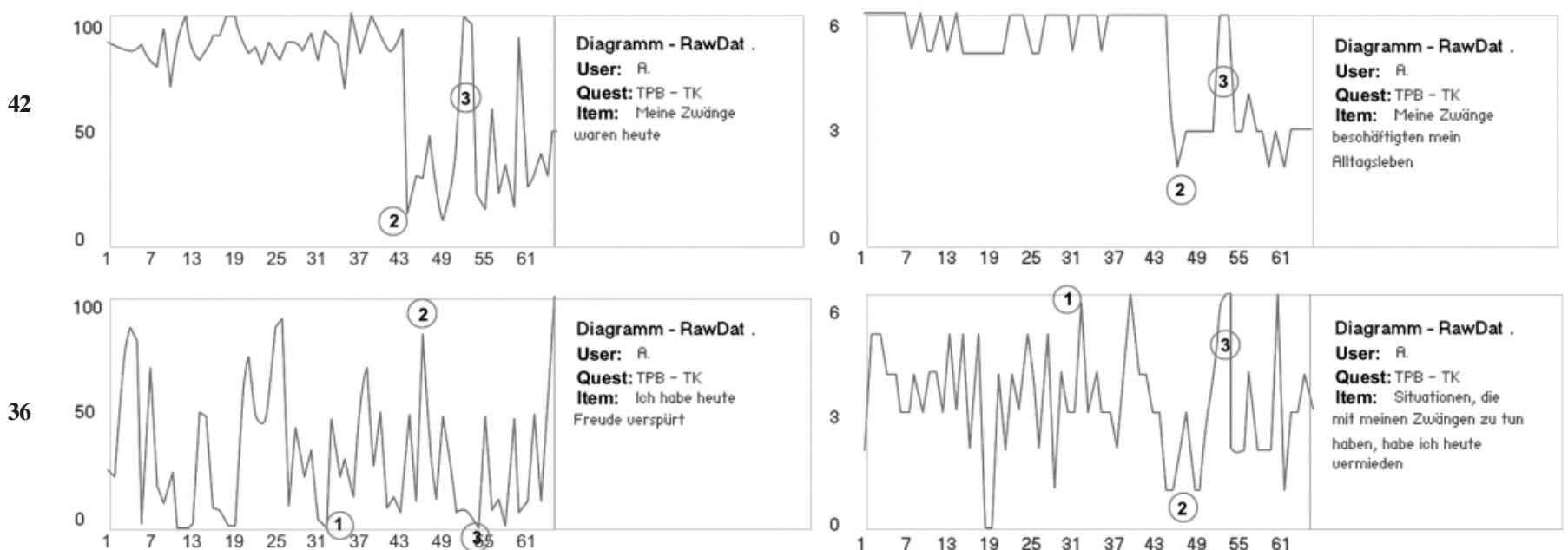

Abb. 1. Verläufe ausgewählter Items des TPB eines Zwangspatienten. Item 21: In der Tagklinik fühle ich mich sicher und unterstützt $(0=$ trifft gar nicht zu, $6=$ trifft voll zu); Item 8: Heute war ich stark motiviert, an meinem Problem bzw. an dessen Lösung zu arbeiten $(0=$ gar nicht, $6=$ sehr stark); Item 35: Mein Selbstwertgefühl war heute $(0=$ sehr gering, $100=$ sehr stark); Item 37: Ich habe heute Scham verspürt $(0=$ gar nicht, $100=$ sehr stark); Item 2: Ich fühle mich jetzt Situationen gewachsen, denen ich mich bisher nicht gewachsen fühlte $(0=$ trifft gar nicht zu, $6=$ trifft voll zu); Item 1: Es wird mir immer besser möglich, meine Probleme aus eigener Kraft zu lösen $(0=$ trifft gar nicht zu, $6=$ trifft voll zu); Item 34 : Ich habe heute Angst verspürt $(0=$ gar nicht, $100=$ sehr stark); Item 45: Heute habe ich mich meinen Zwängen hilflos ausgeliefert gefühlt $(0=$ gar nicht, $6=$ sehr stark $)$; Item 42: Meine Zwänge waren heute $(0=$ sehr gering ausgeprägt, $6=$ sehr stark ausgeprägt); Item 44: Meine Zwänge beschäftigten heute mein Alltagsleben $(0=$ gar nicht, $6=$ sehr stark); Item 36: Ich habe heute Freude verspürt $(0=$ gar nicht, $100=$ sehr stark); Item 46: Situationen, die mit meinen Zwängen zu tun haben, habe ich heute vermieden $(0=$ gar nicht, $6=$ sehr stark $)$. Die Items 34-37 und 42 werden mit visuellen Analogskalen erfasst und in einem Wertebereich von 0-100 dargestellt. Alle anderen Items werden auf einer 7-stufigen Likert-Skala erfasst. Die im Text beschriebenen Ereignisse 1, 2 und 3 sind mit den entsprechenden Ziffern gekennzeichnet. 
Differenzierung, da psychosoziale Konflikte mit Angehörigen häufig durch einen Ortswechsel (z.B. die Aufnahme in eine Klinik) vermieden werden. Durch das tagesklinische Setting und die Möglichkeit der Dateneingabe über einen Heim-PC werden solche Konflikte alltagsnah abgebildet. Das Gleiche gilt für ein ambulantes Setting.

Am Ende des Fragebogens erscheint ein Tagebuchfenster, in das der Patient subjektiv relevante Ereignisse des Tages notiert. So wird retrospektiv erkennbar, was den Patienten am jeweiligen Tag beschäftigt hat und wie sich dies auf die Beantwortung des Fragebogens auswirkt. Auf diese Weise kann die subjektive Relevanz therapeutischer Interventionen, aber auch die Relevanz nichttherapeutischer Ereignisse von Therapeut und Patient besser verstanden werden. Außerdem ermöglichen die Tagebucheinträge ein besseres Verständnis der Kurvenverläufe: Patient und Therapeut können gemeinsam ein mehrwöchiges (oder im ambulanten Setting mehrmonatiges) Zeitfenster auf Kurvenausschläge hin analysieren. Über die Tagebucheinträge erschließen sich kognitive, emotionale und Verhaltensmuster, die bei herkömmlicher Verlaufsdokumentation nicht erkennbar wären, da die subjektive Einschätzung des Patienten nicht synchron dokumentiert wird.

In zahlreichen neueren Publikationen werden die Argumente für ein computerbasiertes ambulantes Assessment systematisch zusammengestellt [z.B. Ebner-Priemer und Sawitzki, 2007; Fahrenberg et al., 2002, 2007].

\section{Behandlungsverlauf eines Patienten mit Sammelzwängen}

Zur Illustration des Prozessmonitorings wählen wir exemplarisch einen Patienten (Herrn A.) mit einer Zwangsstörung, dessen klinisches Bild von Zwangshandlungen und Zwangsgedanken geprägt war (Hort- und Sammelzwang). Zudem litt der Patient unter einer rezidivierenden depressiven Störung. Die Wohnung des 34-jährigen Berufsschullehrers war mit einer Unzahl nutzloser Gegenstände gefüllt und «vermüllte» zunehmend. Gepaart mit einem Kontrollzwang richteten sich seine Befürchtungen darauf, wichtige Gegenstände unabsichtlich wegzuwerfen. Aufgrund des Zustands der Wohnung vermied Herr A. es, andere Menschen in die Wohnung einzuladen, in der er alleine lebte. Auch seine Freundin, zu der er eine ambivalente Beziehung hatte, ebenso wie seine Eltern durften die Wohnung nicht betreten. Er erfand Ausreden und Strategien, um ihnen den Zutritt zur Wohnung zu verwehren. An ein Zusammenziehen mit der Freundin war «aufgrund des Zwangs» nicht zu denken.

Im Rahmen der funktionalen Bedingungsanalyse wurde erkennbar, dass die Kontroll- und Sammelzwänge verschiedene Funktionen erfüllten: (a) Kompensation von Einsamkeits- und Minderwertigkeitsgefühlen, (b) Verhinderung einer emotionalen Ablösung von den Eltern, (c) Vermeidung einer aktiven Freizeitgestaltung, (d) Vermeidung von Nähe in der Partnerschaft und (e) Vermeidung eines Arbeitsplatzwechsels.

Die multimodale Behandlung in der Tagklinik Westend bestand aus täglichen Gruppentherapien, Einzelgesprächen, Gestaltungstherapie, begleiteten Expositionsübungen und einem Home-Flooding (Entrümpeln und Wegwerfen von Gegenständen in der eigenen Wohnung) [Tominschek und Schiepek, 2007].

Herr A. konnte von Anfang an eine gute Beziehung zu den
Therapeuten herstellen und fühlte sich in der Tagesklinik durchgehend sicher und unterstützt. Diese klinische Einschätzung konnte anhand des Items 21 des TPB bestätigt werden (Abb.1). Eine spätere punktuelle Irritation in der therapeutischen Beziehung im Rahmen einer Vertretungssituation manifestierte sich im Kurvenverlauf und konnte mit dem Patienten thematisiert werden.

Das Klima und die soziale Atmosphäre an der Klinik erlebte er nach anfänglicher Unsicherheit zunehmend tragender (wie in den hier nicht dargestellten Verläufen der entschprechenden Faktoren erkennbar). Bei anfänglich hoch ausgeprägter Therapiemotivation (Abb. 1, Item 8) thematisierte er seine Abhängigkeit von den Eltern und der Freundin. Zu dieser Zeit bewegte sich sein Selbstwertgefühl auf einem vergleichsweise niedrigen Level (Abb. 1, Item 35).

Ein entscheidender therapeutischer Ansatzpunkt war das überwältigende Schamgefühl (Abb. 1, Item 37) angesichts des Zustands seiner «zugemüllten» Wohnung, das wir erst über die Analyse der im TPB abgefragten Emotionen identifizieren konnten. Als wir den Patienten auf seine Selbsteinschätzung ansprachen, erzählte er uns, dass vergebliche Aufräumversuche in den letzten Jahren zu Gefühlen von Demoralisierung und Resignation geführt hatten. Herr A. fühlte sich antriebslos und erschöpft. Ein vergleichbares Selbsterleben wiederholte sich am 32. Therapietag (in Abb. 1 mit der Ziffer 1 gekennzeichnet). Trotz positiven Therapieverlaufs erlebte er einen dramatischen emotionalen und motivationalen Einbruch (Abb. 1, Items 8, 35-37, 45, 46), als es in der Therapie darum ging, die Expositionsübungen für das Heim-Flooding zu konkretisieren. Durch eine Analyse seiner therapeutischen Entwicklung anhand der visualisierten Zeitreihen und Tagebucheintragungen, welche seine Kompetenzen und Stärken verdeutlichten, konnte diese Krise schnell relativiert und überwunden werden.

Der Patient formulierte einen Flooding-Vertrag und setzte sich intensiv mit bevorstehenden Übungen auseinander (34.42. Therapietag). Am ersten Tag der Reizüberflutung (Flooding) (44. Therapietag, in Abb. 1 jeweils mit der Ziffer 2 markiert) ging der Patient nach mehrjähriger Vermeidung in einer begleiteten Übung mit einer Co-Therapeutin der Tagesklinik in seine Wohnung. Sein Schamgefühl war an diesem Tag auf einem Maximalwert (Abb. 1, Item 37), und auch seine Angst war sehr stark ausgeprägt (Abb. 1, Item 34). Gleichzeitig wird erkennbar, dass dieser entscheidende Schritt sein Selbstwertgefühl stärkt (vgl. den Peak seines Selbstwertgefühls kurz nach dem Tag des Flooding-Beginns; Abb. 1, Item 35). Nach diesem Ereignis sieht er sich besser in der Lage, seine Probleme aus eigener Kraft zu lösen (Abb. 1, Item 1) und fühlt sich jetzt Situationen gewachsen, denen er sich bisher nicht gewachsen fühlte (Abb. 1, Item 2). Er erlebt einen sprunghaften Zugewinn an Selbstwirksamkeit und Selbstvertrauen. Auch seine Freude kurz nach Beginn des Floodings (d.h. nachdem er bereits in wenigen Tagen Effekte erkennt) macht einen Sprung (Abb. 1, Item 36). Die Effekte sind in der Tat unmittel- 

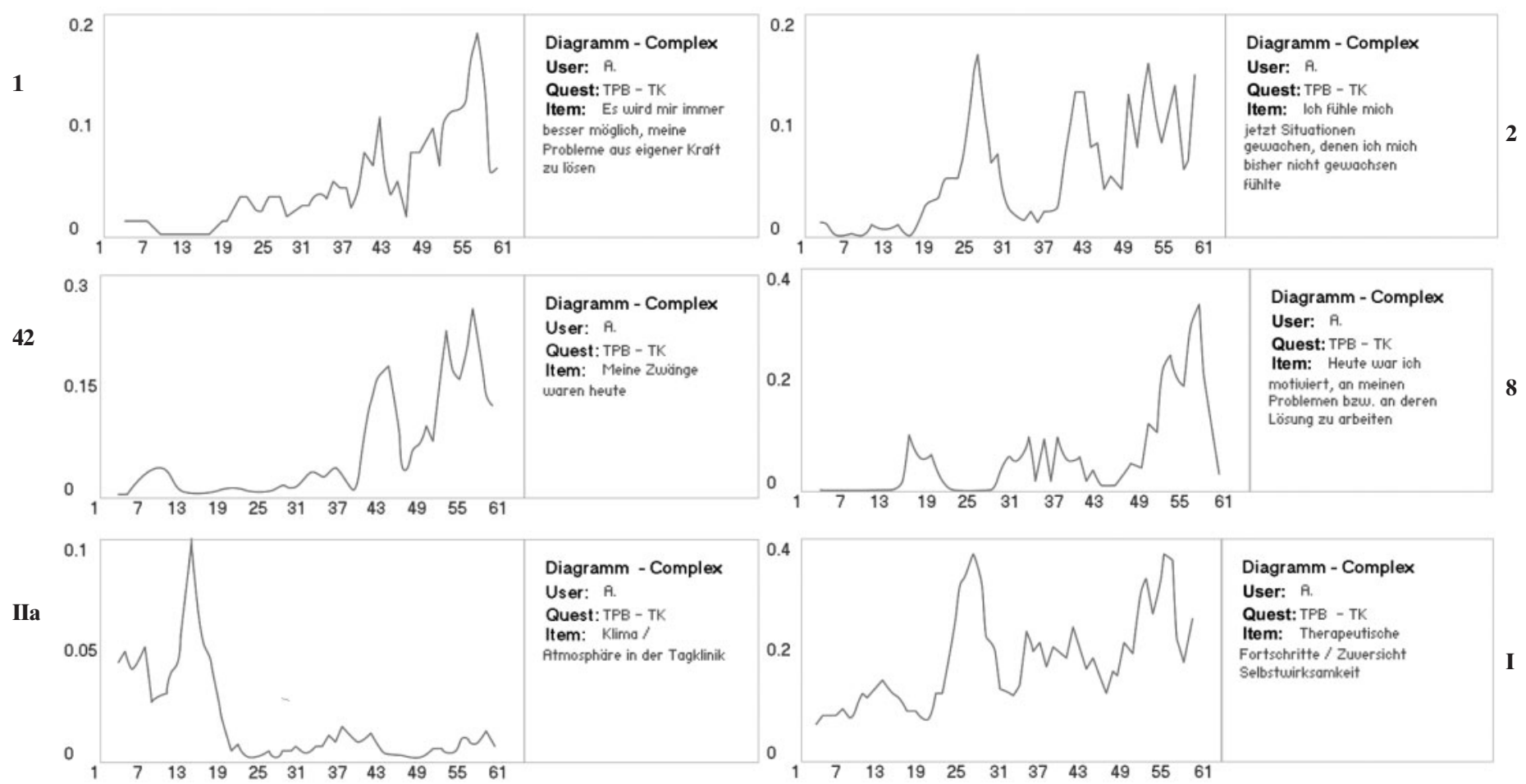

Abb. 2. Die Entwicklung der dynamischen Komplexität der Items 1, 2, 42 und 8 sowie der Faktoren IIa (Klima / Atmosphäre in der Tagklinik) und I (Therapeutische Fortschritte, Zuversicht / Selbstwirksamkeit). Dieses Komplexitätsmaß wurde in einem Gleitfenster von 7 Messpunkten Breite berechnet. Daher sind die Graphen dieses Kennwerts um 6 Punkte kürzer als die der Rohwerte und Faktoren.

bar erkennbar: Nach Beginn des Floodings erlebt er sich seinen Zwängen weniger hilflos ausgeliefert (Abb. 1, Item 45), und die zunächst sehr starke Angst reduziert sich (Abb. 1, Item 34). Die Ausprägung der Zwänge (Abb. 1, Item 42) reduziert sich ebenso drastisch und tritt auch im Alltagsleben in den Hintergrund (Abb. 1, Item 44). Schließlich reduziert sich auch der Grad der Vermeidung (Abb. 1, Item 46).

Die Zeitreihen wurden mit dem Patienten auch während der Flooding-Phase besprochen und die Therapieschritte differenziert reflektiert, was ihm die Möglichkeit gab, die verschiedenen Aspekte der erlebten Situationen nochmals zu betrachten und aufgrund der erstarkten Selbstwirksamkeit und der verringerten Hilflosigkeit ein positiveres Selbstwertgefühl aufzubauen. Auch dies half, seine intensiven Schamgefühle zu reduzieren.

Dennoch zeigt sich die Situation während der Flooding-Phase keineswegs kontinuierlich stabil. Insbesondere die Therapietage 52 und 53 waren von einer Krise geprägt (in Abb. 1 mit der Ziffer 3 gekennzeichnet). Auslöser waren weniger äußere Ereignisse als vielmehr die Erschöpfung des Patienten. Er fiel in sein altes Vermeidungsmuster zurück (Items 42, 44 und 45), sein Optimismus und die erlebte Selbstwirksamkeit brachen ein. Die unmittelbare Reflexion des Verlaufs gemeinsam mit dem Patienten anhand der Zeitreihen führte auch hier wieder zu einer Unterbrechung des Vermeidungsverhaltens und machte die Fortführung der Expositionsübungen möglich. Die angeleitete und ressourcenorientierte Auseinandersetzung mit seiner bisherigen Entwicklung trug dazu bei, seine Motivation wieder aufzubauen. Auch in anderen Fällen konnte beobachtet werden, dass ein datenbasiertes Feedback zur Therapieentwicklung motivationsfördernd wirkt.

Man erkennt an zahlreichen Verläufen, dass der Beginn des Floodings zwar zu einem Sprung in der Entwicklung geführt, aber auch eine Phase dramatischer Instabilität eingeleitet hat, z.B. hinsichtlich der Therapiemotivation (Abb. 1, Item 8), der Selbstwirksamkeit (Abb. 1, Items 1 und 2), der Ausprägung der Zwänge und des Vermeidungsverhaltens (Abb. 1, Items 42, 44 und 46). Abbildung 2 zeigt für einige Items und Faktoren die Entwicklung der dynamischen Komplexität, ein Maß, das für solche Instabilitäten besonders sensitiv ist. Dargestellt sind die Items 1, 2, 8 und 42 sowie die Faktoren IIa und I. Auffällig und für Psychotherapieverläufe durchaus charakteristisch ist die gegenläufige Entwicklung dieser Faktoren: Nachdem die anfängliche Instabilität im erlebten sozialen Milieu fast komplett reduziert ist (Faktor IIa), entfaltet sich die Dynamik im Therapiegeschehen (Faktor I). Bemerkenswert ist die Tatsache, dass Ordnungsübergänge (wie in diesem Fall am Beginn des Floodings) oft mit kritischen Instabilitäten in der quantitativ messbaren Dynamik und gleichzeitig mit intensiv erlebter emotionaler Ambivalenz und Ambiguität einhergehen. Wie oben beschrieben erlebte Herr A. starke Angst und Scham, aber auch Optimismus, Zuversicht und ein Gefühl von Selbstwirksamkeit, als er zum ersten Mal seit Jahren mit einer anderen Person seine Wohnung aufsuchte. 
Bei Entlassung hatte sich die Zwangssymptomatik des Patienten - erfasst mit der Y-BOCS [Hand und Büttner-Westphal, 1991] in wöchentlichem Abstand - deutlich gebessert, von 27 bei Aufnahme (schwere Zwangssymptomatik) auf 14 (leichte Zwangssymptomatik). Der Patient hatte eine kritische Auseinandersetzung mit den Eltern geführt, war deutlich selbständiger in der Lebensführung und pflegte nun wieder Sozialkontakte. Die starren Muster seines Zwangs und Rückzugsverhaltens waren aufgebrochen und destabilisiert, die Resultate des Monitorings zeigen aber, dass die neuen Verhaltensmuster im Rahmen einer ambulanten Weiterbehandlung stabilisiert werden müssen.

Zusammenfassend ist festzustellen, dass das therapeutische Setting und der Kontakt zu Therapeuten und Mitpatienten die notwendige Stabilität für die inhaltliche psychotherapeutische Auseinandersetzung geschaffen haben. Dies bildet sich in den Kurvenverläufen ab.

Bei der Besprechung der Kurvenverläufe können unterschiedliche Aspekte thematisiert werden:

- Welche Erfolge und Misserfolge erlebte der Patient seit dem letzten Termin?

- Welche Strategien, Stärken und Kompetenzen konnte er einsetzen?

- Sind für bestimmte Ereignisse (z.B. starke Symptombelastung, ausgeprägte negative Emotionen) innere oder äußere Bedingungen oder Auslöser zu erkennen? Wiederholen sich bestimmte Verhaltensmuster einschließlich ihrer kognitiven und emotionalen Komponenten? (Damit kann das RealTime-Monitoring zur Erstellung oder Validierung funktioneller Bedingungsanalysen beitragen.)

- Wie erlebt der Patient die Entwicklung seiner Selbstwirksamkeit, seiner Therapiemotivation, seiner Fortschritte, seines Problemverständnisses usw.?

- Erlebt der Patient seinen Behandlungskontext hinreichend stabil, z.B. hinsichtlich Setting, Struktur, Therapiebeziehung, um neue Schritte im Veränderungsprozess zu riskieren (z.B. Expositionsübungen, Verhaltensexperimente)?

- Welche Emotionen und Emotionsschwankungen (z.B. bei Borderline-Störungen) sind für den Patienten charakteristisch?

- Werden kritische Phasen von positiven Erwartungen, Interesse am Geschehen und von einem Gefühl der Selbstwirksamkeit getragen?

Mit Blick auf das Feedback können auch Themen angesprochen werden, die vielleicht sonst nicht berücksichtigt würden (z.B. Konflikte mit den Mitpatienten, angstbesetzte Inhalte). Vor allem zeitlich wiederkehrende Muster lassen sich feststellen, die bei einer Querschnittsbetrachtung nicht auffallen würden.

\section{Diskussion}

Das vorliegende Fallbeispiel macht deutlich, dass der Therapieverlauf mit Hilfe von täglichen Selbsteinschätzungen und daraus resultierenden Zeitreihen differenziert abgebildet werden kann. Hinzu kommen die täglichen Einträge des Patienten in sein elektronisches Tagebuch, welche die quantitativen Verläufe mit qualitativen Informationen bereichern. Beides zusammen dient als Grundlage für regelmäßige Besprechungen des Therapieverlaufs und hilft dem Patienten wie auch dem Therapeuten, den Therapieverlauf besser zu verstehen. Besonderen Stellenwert gewinnt dies bei der Therapie von persönlichkeitsgestörten Patienten, die ja ihr sozial unverträgliches Verhalten häufig als angemessen (ich-synton) erleben.

Die inhaltliche Auseinandersetzung mit drängenden Fragestellungen (Trennung vom Partner, Ablösung von den Eltern, Arbeitsplatzwechsel usw.) und das Expositionstraining führten bei unserem Patienten zu einer inneren Destabilisierung, die der Patient ambivalent erlebte: Einerseits wünschte er sich eine Veränderung, andererseits hatte er Angst vor den Risiken. Würde er alleine zurechtkommen, neue Bezugspersonen oder einen anderen Arbeitsplatz finden, die damit verbundenen Gefühle aushalten können? Solche Ambivalenzen werden bei Ordnungsübergängen besonders spürbar. Bei Zwangspatienten ist ein solcher Ordnungsübergang häufig der Beginn des Floodings. Sie treffen die Entscheidung, sich auf eine massive Reizkonfrontation einzulassen und nehmen die damit verbundene emotionale Belastung in Kauf. Gleichzeitig können in dieser Phase bisher verdrängte oder verleugnete Konflikte bewusst und somit einer psychotherapeutischen Bearbeitung zugänglich werden.

Über Prozessdiagnostik und Prozessfeedback in der Praxis hinaus dient das «Synergetic Navigation System» als Forschungsinstrument. Die kombinierte Untersuchung von Therapieeffekten und Therapieverläufen kann damit auf eine breite Datenbasis gestellt werden. War noch vor wenigen Jahrzehnten und im Grunde bis heute intensive Prozessforschung gleichzusetzen mit Einzelfallforschung [vgl. Petermann, 1979], so können nun Datenbanken mit großen Fallzahlen angelegt werden, um Prozesse bei unterschiedlichen Diagnosen und Patientenmerkmalen, verschiedenen Therapiemethoden und -settings, bei unterschiedlich erfolgreichen Behandlungen oder Abbrüchen sowie mit unterschiedlichen dynamischen Merkmalen zu untersuchen. Eine solche Datenbank befindet sich in Vorbereitung.

Das «Synergetic Navigation System» dient auch zur Erzeugung von psychologischen Korrelaten neurobiologischer Veränderungen bei Psychotherapien und zur Feststellung geeigneter Zeitpunkte für die Durchführung von Messungen mit funktioneller Magnetresonanztomographie (fMRT) [Schiepek und Schönfelder, 2007]. Hierbei konnten interessante Korrespondenzen zwischen der Veränderung neuronaler Aktivierungsmuster und dem Auftreten therapeutischer Ordnungsübergänge im Sinne der Synergetik gefunden werden [Schiepek et al., im Druck]. Ein aktuelles Forschungsprojekt hierzu wird derzeit in Zusammenarbeit mit der Tagklinik Westend, der Psychosomatischen Klinik Windach, dem Center für Complex Systems, der Klinik für Psychiatrie und Psychotherapie der Universität München (LMU) und der Medizinuniversität Wien durchgeführt. 


\section{Literatur}

Asay TP, Lambert MJ, Gregersen AT, Goates MK Using patient-refocused research in evaluating treatment outcome in private practice. J Clin Psychol 2002;58:1213-1225.

Clarkin JF, Levy KN: The influence of client variables on psychotherapy; in Lambert MJ (ed): Bergin and Garfield's Handbook of Psychotherapy and Behavior Change. New York, Wiley, 2004.

Ebner-Priemer, Sawitzki G: Ambulatory assessment of affective instability in borderline personality disorder. Europ J Psychol Assessment 2007;23:238-247.

Fahrenberg J, Leonhart R, Foerster F: Alltagsnahe Psychologie. Datenerhebung im Feld mit hand-held PC und physiologischem Mess-System. Bern, Huber, 2002. Fahrenberg J, Myrtek M, Pawlik K, Perrez M: Ambulantes Assessment - Verhalten im Alltagskontext erfassen. Psychol Rundsch 2007;58:12-23.

Haken H: Brain Dynamics. Berlin, Springer, 2002.

Haken H: Synergetik der Gehirnfunktionen; in Schiepek G (Hrsg): Neurobiologie der Psychotherapie. Stuttgart, Schattauer, 2003.

Haken, H: Synergetics. Introduction and Advanced Topics. Berlin, Springer, 2004.

Haken H, Schiepek G: Synergetik in der Psychologie. Selbstorganisation verstehen und gestalten. Göttingen, Hogrefe, 2006.

Hand I, Büttner-Westphal H: Die Yale-Brown Obsessive Compulsive Scale (Y-BOCS): Ein halbstrukturiertes Interview zur Beurteilung des Schweregrades von Denk- und Handlungszwängen. Verhaltenstherapie 1991;1:223-255.

Heuft G, Senf W: Praxis der Qualitätssicherung in der Psychotherapie: Das Manual zur Psy-BaDo. Stuttgart, Thieme, 1998.
Kowalik ZJ, Schiepek G, Kumpf K, Roberts LE, Elbert T: Psychotherapy as a chaotic process II: The application of nonlinear analysis methods on quasi time series of the client-therapist-interaction: A nonstationary approach. Psychother Res 1997;7:197-218.

Lambert MJ, Hansen NB, Finch AE: Patient-focused research: Using patient outcome data to enhance treatment effects. J Consult Clin Psychol 2001a;69:159-172. Lambert MJ, Whipple JL, Smart DW, Vermeersch DA, Nielsen SL, Hawkins EJ: The effects of providing therapists with feedback on patient progress during psychotherapy: Are outcomes enhanced? Psychother Res 2001b;11:49-68.

Lambert MJ, Whipple JL, Vermeersch DA, Smart DW, Hawkins EJ, Nielsen SL, Goates M: Enhancing psychotherapy outcomes via providing feedback on client progress: A replication. Clin Psychol Psychother 2002; 9:91-103.

Lambert MJ, Ogles BM: The efficacy and effectiveness of psychotherapy; in Lambert MJ (ed): Bergin and Garfield's Handbook of Psychotherapy and Behavior Change. New York, Wiley, 2004.

Orlinsky DE, Ronnestad MH, Willutzki U: Fifty years of psychotherapy process-outcome research: Continuity and change; in Lambert MJ (ed): Bergin and Garfield's Handbook of Psychotherapy and Behavior Change. New York, Wiley, 2004.

Percevic R, Gallas C, Arikan L, Mößner M, Kordy H: Internet-gestützte Qualitätssicherung und Ergebnismonitoring in Psychotherapie, Psychiatrie und psychosomatischer Medizin. Psychotherapeut 2006;51:395397.

Petermann F (Hrsg): Einzelfallforschung. München, Urban und Schwarzenberg, 1979.
Popovych OV, Hauptmann C, Tass PA: Effective desynchronization by nonlinear delayed feedback. Phys Rev Lett 2005;94:164102/1-164102/4.

Popovych OV, Hauptmann C, Tass PA: Control of neural synchrony by nonlinear delayed feedback. Biol Cybern 2006;95:69-85.

Schiepek G, Kowalik ZJ, Schütz A, Köhler M, Richter K, Strunk G, Mühlnickel W, Elbert T: Psychotherapy as a chaotic process I. Coding the client-therapist-interaction by means of sequential plan analysis and the search for chaos: A stationary approach. Psychother Res 1997;7:173-194.

Schiepek G, Weihrauch S, Eckert H, Trump T, Droste S, Picht A, Spreckelsen C: Datenbasiertes RealTime Monitoring als Grundlage einer gezielten Erfassung von Gehirnzuständen im psychotherapeutischen Prozess; in Schiepek G (Hrsg): Neurobiologie der Psychotherapie. Stuttgart, Schattauer, 2003.

Schiepek G, Schönfelder V: Musterhafter Wandel. Gehirn und Geist 2007;10:52-58.

Schiepek G, Tominschek I; Karch S, Lutz J, Mulert C, Meindl T, Pogarell O: A controlled single case study with repeated fMRI measures during the treatment of a patient with obsessive-compulsive disorder: Testing the nonlinear dynamic approach to psychotherapy. World J Biol Psychiatry 2008, in press.

Singer W, Gray CM: Visual feature integration and the temporal correlation hypothesis. Ann Rev Neurosci 1995;18:555-586.

Strunk G, Schiepek G: Systemische Psychologie. Eine Einführung in die komplexen Grundlagen menschlichen Verhaltens. Heidelberg, Spektrum , 2006.

Tominschek I, Schiepek G: Zwangsstörungen. Ein systemisch-integrativer Behandlungsansatz. Göttingen, Hogrefe, 2007. 